\title{
BMJ Open National mixed methods evaluation of the effects of removing legal barriers to full practice authority of Dutch nurse practitioners and physician assistants
}

\author{
Daisy P De Bruijn-Geraets, ${ }^{1,2}$ Yvonne J L van Eijk-Hustings, ${ }^{1,2}$ \\ Monique C M Bessems-Beks, ${ }^{1}$ Brigitte A B Essers, ${ }^{1,2}$ Carmen D Dirksen,, ${ }^{1,2}$ \\ Hubertus Johannes Maria Vrijhoef $f^{1,3,4}$
}

To cite: De Bruijn-Geraets DP, van Eijk-Hustings YJL, BessemsBeks MCM, et al. National mixed methods evaluation of the effects of removing legal barriers to full practice authority of Dutch nurse practitioners and physician assistants. BMJ Open 2018;8:e019962. doi:10.1136/ bmjopen-2017-019962

- Prepublication history and additional material for this paper are available online. To view these files, please visit the journal online (http://dx.doi. org/10.1136/bmjopen-2017019962).

Received 10 0ctober 2017 Revised 10 May 2018 Accepted 14 May 2018

Check for updates

'Department of Patient and Care, Clinical Epidemiology and Medical Technology Assessment Maastricht University Medical Centre, Maastricht, The Netherlands

${ }^{2}$ School CAPHRI, Care and

Public Health Research

Institute, Maastricht University,

Maastricht, The Netherlands

${ }^{3}$ Panaxea, Amsterdam, The

Netherlands

${ }^{4}$ Department of Primary Care,

Vrije Universiteit Brussel,

Brussels, Belgium

Correspondence to

Daisy P De Bruijn-Geraets

daisy.de.bruijn@mumc.nl

\section{ABSTRACT}

Objective To evaluate the effects of granting legal full practice authority (FPA) to nurse practitioners (NP) and physician assistants $(\mathrm{PA})$ regarding the performance of specified reserved medical procedures and to support governmental decision-making.

Design Nationwide mixed methods design with triangulation of quantitative (Pre-post test design) and qualitative data (expert interviews and focus groups). Methods Surveys focused on the performance of the procedures (monthly number, authorisation mode, consultations and procedural time) and legal crosscompliance requirements (adherence with protocols, competence). Interviews focused on competence, knowledge, skills, responsibilities, routine behaviour, NP/PA role, acceptance, organisational structure, collaboration, consultation, NP/PA positioning, adherence with protocols and resources. Data collection took place between 2011 and 2015.

Results Quantitative data included 1251 NPs, 798 PAs and 504 physicians. Besides, expert interviews with 33 healthcare providers and 28 key stakeholders, and 5 focus groups ( 31 healthcare providers) were held. After obtaining FPA, the proportion of NPs and PAs performing reserved procedures increased from $77 \%$ to $85 \%$ and from $86 \%$ to $93 \%$, respectively; the proportion of procedures performed on own authority increased from $63 \%$ to $76 \%$ for NPs and from $67 \%$ to $71 \%$ for PAs. The mean number of monthly contacts between NPs/PAs and physicians about procedures decreased (from 81 to 49 and from 107 to 54 , respectively), as did the mean duration in minutes (from 9.9 to 8.6 and from 8.8 to 7.4 , respectively). Utilisation of FPA was dependent on the setting, as scepticism of physicians and medical boards hampered full implementation. Legal cross-compliance requirements were mostly fulfilled.

Conclusions Informal practice was legalised. The opportunities to independently perform catheterisations, injections, prescribing, punctures and small surgical procedures were highly used. Care processes were organised more efficiently, services were performed by the most appropriate healthcare provider and conditions were met. This led to the recommendation to continue with FPA.
Strengths and limitations of this study

- This is the first study to systematically evaluate the effects of introducing legal full practice authority on processes and outcomes of care.

- This study offers a novel mixed methods approach for evaluation as well as data for cross-national comparison.

- The use of a mixed methods design with triangulation provides a comprehensive insight into a complex, sensitive subject.

- The limitations of this study, mostly anticipated by triangulation, include a moderate participation rate and the restraints of causal interference in the One Group Pre-post test design with quantitative data.

- The novel survey tool was not tested for reliability and validity.

\section{INTRODUCTION}

Despite task shifting being a common strategy for healthcare reform in many countries, its regulation does not keep pace. Task shifting concerns the redistribution of tasks among health workforce teams. It is no longer exclusively applied to anticipate health workers shortage, specifically physicians, although the benefits it has on the quality of healthcare have been recognised. ${ }^{1-4}$ In particular, nurse practitioners (NP) and physician assistants (PA) are well-qualified healthcare providers, who can comply with the requirements of healthcare reform, but practising to the full extent of their education and training (full practice authority: FPA) is often hampered by (the absence of) regulation. ${ }^{5-7}$ In a review on task shifting from physicians to advanced nurses in five Western countries, three levels of regulations have been distinguished: national regulation, decentralised regulation and unregulated, setting-dependent governance. ${ }^{8}$ Both level and content of the 
regulation varied by country. Authority to prescribe medication was regulated by law in all countries, but the level of independence varied. The authors concluded that regulation for task shifting can either act as a potential barrier (when restrictive in nature) or be enabling (when up to date with educational competencies). Hence, for task shifting to be effective, regulation is decisive.

In the Netherlands, the Ministry of Health, Welfare and Sports (HWS) has adopted national task shifting policy. One measure to advance task shifting is to grant FPA to NPs and PAs. The number of registered NPs and PAs at present is over $3000^{9}$ and $950,{ }^{10}$ respectively, most being employed in general and academic hospitals, general practitioner practices, mental healthcare institutions and nursing homes. ${ }^{11}$ Both NPs and PAs work at a master degree level. NPs work in medical and nursing domains, often with a specific group of patients. PAs only work in the medical domain. This involves both tasks in (in) direct patient care like consultations, visits, case management and file-keeping, as well as not patient-related tasks like development of integrated care, quality improvement programmes and protocols; training of other care providers and management tasks. In direct patient care, within the agreed working domain, NPs and PAs independently come to a (differential) diagnosis based on self-initiated anamnesis, physical and/or psychiatric examination and additional diagnostics and apply evidence-based interventions. ${ }^{12} 13$

In doing so, NP/PA autonomy (and thus optimal use of their competencies) was hampered by a legally required physician's consent to perform certain medical procedures, as described in the Individual Health Care Professions Act (in Dutch Wet BIG). In this law, the performing of specified medical procedures, termed reserved procedures, is reserved for defined healthcare professionals. By means of an amendment, followed by two Orders in Council in 2012, NPs and PAs have been legally authorised to independently indicate, execute and delegate the following reserved procedures: catheterisation, cardioversion, defibrillation, endoscopy, injection, puncture, prescribing prescription-only medicines and simple surgical procedures, creating truly FPA. NPs and PAs must be competent to perform these procedures and work according to guidelines containing cooperation agreements with physicians (ie, protocols) including the range of prescriptive authority, a formulary may be added. The Orders in Council are valid for a period of 5 years and are subject to evaluation. Commissioned by the Ministry of HWS, we carried out this evaluation to support decision-making regarding continuation of the temporary practice authorities of NPs and PAs.

\section{OBJECTIVE}

We aimed to systematically evaluate the effects of granting FPA to NPs and PAs on the processes and outcomes of care. Due to the elimination of required consent, we hypothesised that NPs/PAs would perform more procedures on their own authority and that procedure times would become shorter overall. Furthermore, we expected legal cross-compliance requirements would be met.

\section{METHODS}

The study protocol, including a comprehensive description of the methods, has been published elsewhere. ${ }^{14}$ The study meets the STROBE ${ }^{15}$ and COREQ ${ }^{16}$ criteria for reporting quantitative and qualitative study results.

\section{Design}

This study used a mixed methods design with a concurrent triangulation strategy (table 1). Quantitative (surveys) and qualitative (interviews and focus groups) data were collected, analysed and interpreted in the same time frame, and were considered of equal importance. ${ }^{17-20}$ Quantitative data were collected according to a One Group Pre-post test design with three time points: before the amended law (T0) and one (T1) and two and a half years (T2) postamendment. As the amendment affects the entire country, selection of a control group was impossible.

Both methods were based on existing theoretical frameworks. The quantitative piece was based on the Nursing Role Effectiveness framework of Sidani and Irvine ${ }^{21-23}$ combined with Donabedian's model ${ }^{24}$ for assessing healthcare quality and proposing specific relationships between structures, processes and outcomes. The qualitative piece was based on the implementation model of Grol and Wensing. ${ }^{25}$ Factors that could affect the implementation process were categorised as individual health professionals' characteristics and social, organisational and societal components conforming to the model.

Triangulation was carried out according to the transformation model,${ }^{18}$ in which qualitative data were quantified and quantitative data were converted into a narrative.

\section{Participants and recruitment}

We collected quantitative data (figure 1) from a purposive sample of registered NPs and graduated PAs working in the Netherlands. Professional organisations, applied universities with NP/PA programmes and national NP/ PA congress committees all assisted in data collection by means of newsletters, websites and direct contact. Every $\mathrm{NP} / \mathrm{PA}$ was asked to invite two collaborating physicians for participation. Qualitative data were collected through semistructured expert interviews and focus groups. Supported by the above organisations and facilities, we recruited NPs/PAs, physicians (as case studies) and stakeholders (including management staff across all levels in various settings, professional medical organisations, professional trainers, the Health Care Inspectorate, and Netherlands Institute for Accreditation in Healthcare) for expert interviews, taking into account the broad distribution of health organisation types and medical specialisations, as well as geographical coverage. Case studies across the country were also invited to focus group meetings. 
Table 1 Triangulation of structure, process and outcome measures

\section{Structure}

Quantitative data

NP/PA, physician background characteristics

Job title, specialism, age, gender, education, years of experience

Qualitative data

NP/PA, physician background

characteristics

Job title, specialism, gender

Organisation background characteristics

Organisation background characteristics

Type organisation, specialism, urbanisation level

Type organisation, specialism

\section{Collaboration}

Presence of collaborating physicians, availability of supervising physicians,

satisfaction (5-point Likert) about this

\section{Collaboration}

\section{Positioning NP/PA}

Resources

\section{Processes}

\section{Quantitative data}

Adherence with protocols

Presence, contents and enforcement of protocols ${ }^{26}$

\section{Consultation}

Frequency and mode of consultations ${ }^{26}$ and sufficiency of this

Competence Competence

Appraisal of competence ${ }^{27}$

\section{Qualitative data}

Adherence with protocols

\section{Consultation}

Competence

Role

Routine behaviour

Knowledge/skills

Acceptance

\section{Outcomes}

\section{Quantitative data}

\section{Appropriate performance}

Monthly performance of specified reserved procedures and authorisation

Qualitative data

mode. For prescribing medicines: distinction between new, refill and change in dosage prescriptions; name and dosage of the drug. For injections: distinction between joints, tendon sheaths and keloids; administration of sclerotherapy; administration of local anaesthetics; intramuscular, intravenous, subcutaneous and intracardiac injections and name and dosage of the drug

\section{Costs}

Duration of specific reserved procedures including consultation and process

time, number and duration of intercollegial consultation.

NP, nurse practitioner; PA, physician assistant.

\section{Data collection}

For the quantitative data collection at T0, T1, T2 (table 1), three different web-based and paper-based questionnaires were used. ${ }^{14}$ The first questionnaire, the quick scan, consisted of five short questions and directed a maximal response. NPs and PAs could register the reserved procedures (main group) they had performed at a given time, complemented with the type of care organisation and medical specialism. By means of the NP/PA and physician questionnaire (table 1), data were collected on the estimated number of procedures performed monthly (including whose authorisation was required and whether consultation with a physician was needed) and the procedural time needed (including consultation/assignment time). Background characteristics (ie, age, gender, job title, specialism, years of experience and care setting) and the legal cross-compliance requirements adherence to protocols ${ }^{26}$ and competence ${ }^{27}$ were also asked.

Expert interviews and focus groups were all directed by interview guides, which were developed in consultation with the study advisory board based on the implementation model (table 1). ${ }^{25}$ Expert interviews were conducted by author DPDBG. YJLvEH moderated the focus group interviews, with DPDBG and MCMBB acting as observers. The independent interviewers had ample previous experience in conducting interviews. Prior to the interviews, the interviewers introduced themselves (stating their personal goals) and informed the participants about the general objectives of both the study and interview. Afterwards, an oral provisional summary was provided. The 


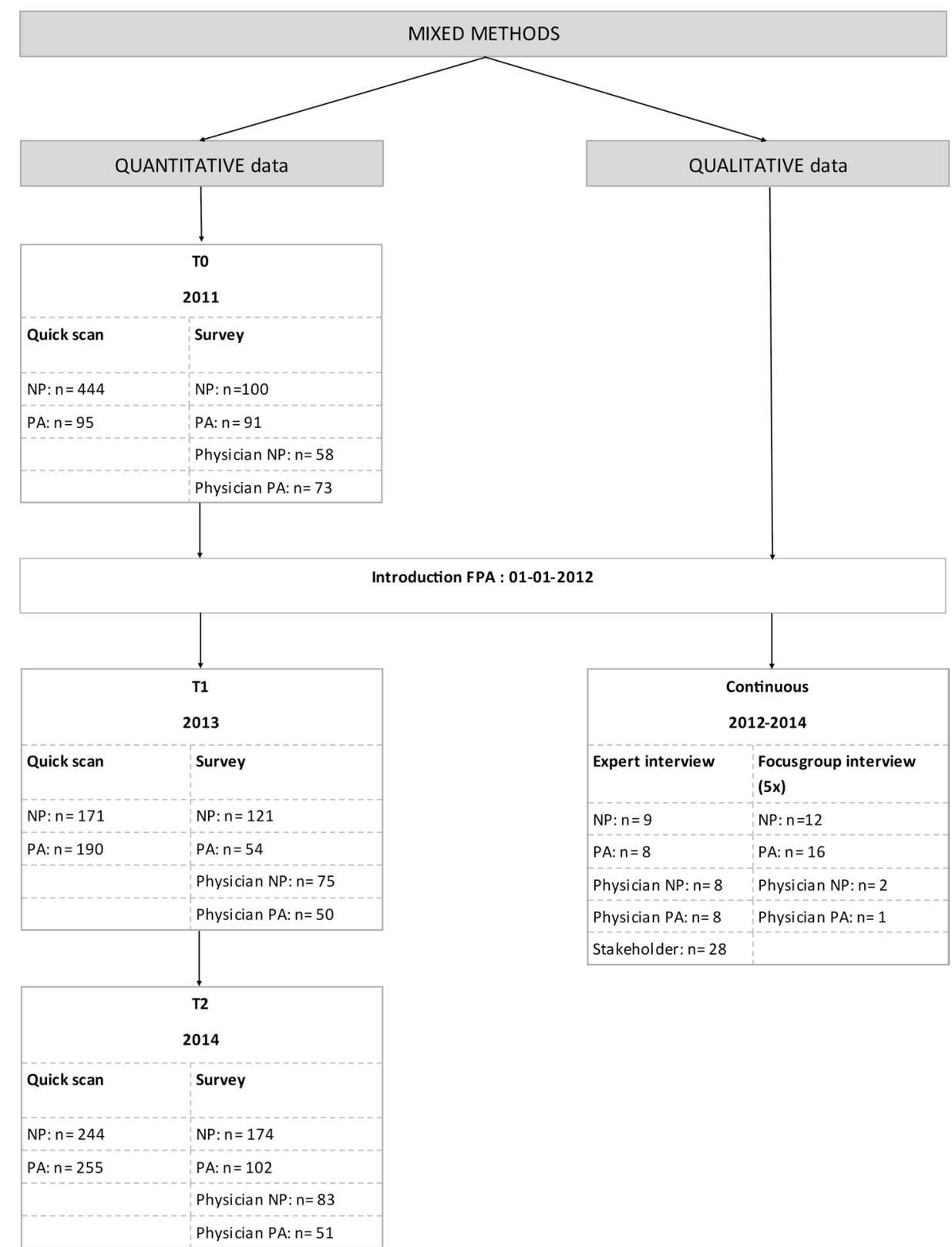

Figure 1 Study flow. FPA, full practice authority; NP, nurse practitioner; PA, physician assistant; physician NP, physician collaborating with an NP; physician PA, physician collaborating with a PA; stakeholders, management staff across all levels in various settings, professional (medical) organisations, professional trainers, pharmacies in various settings, general practitioner (GP) out-of-office service, the Health Care Inspectorate, Netherlands Institute for Accreditation in Healthcare and health insurance companies.

expert interview guide was pilot-tested over three interviews. After each quantitative data time point and analysis, the interview protocol was reviewed and refined. At first, open questions were raised about the main factors of the implementation model. A checklist of derived key elements was kept. When the conversation did not elicit responses about these elements, direct questions were asked. The one-on-one interviews were conducted in person (or by phone) at a time and location that was convenient for the interviewee. Interviews lasted approximately 20-40 min for case studies and $45-60 \mathrm{~min}$ for stakeholders.
In the focus group guide, four central themes were drawn up to elicit multiple perspectives: the situation in which NPs/PAs performed reserved procedures, facilitators and barriers for that situation and preconditions for FPA. The themes were also compared with the factors in the implementation mode ${ }^{25}$ and unanticipated or vague elements were brought up later by the observers who made extensive field notes. All participants completed a written form providing demographic information. The focus group interviews (2 hours each) were conducted at four different meeting centres across the Netherlands. 
The expert interviews and focus groups were digitally recorded and transcribed verbatim. The researchers checked the transcriptions and abstracts were sent to the participants for verification (ie, member check).

\section{Statistical analysis}

Quantitative data analysis was performed using SPSS software V.22. ${ }^{28} \mathrm{~T} 1$ was considered to be a mid-term evaluation (values are presented but not tested). For continuous variables, means with the corresponding $95 \%$ CI were calculated and in case of non-normality (determined with the Kolmogorov-Smirnov normality test), medians and IQRs were used. For the number and duration of monthly performed procedures, extreme values $(>5$ SDs from the mean) were excluded. Before determining the mean monthly performance for the main groups of reserved procedures (eg, catheterisation), numbers from the separate procedures (eg, bladder catheterisation) were added up for each respondent. The original plan was to perform longitudinal group analyses. ${ }^{29}$ However, as the composition of the groups at time points strongly diverged (only $12 \%$ of the NPs/PAs completed the surveys at each time point, $66 \%$ at a single one), the Mann-Whitney test was instead used to test for differences between T0 and T2. For categorical variables, frequencies and percentages were calculated and the $\mathrm{X}^{2}$ test (with $1 \mathrm{df}$ ) was used to test for differences between T0 and T2. Tests were performed at both group and subgroup levels for participants taking part in T0 and T2. Only group data are presented, although differences for subgroup data are discussed in the main text. All tests were performed two sided with a $\mathrm{p}$ value of $>0.05$.

The editing analysis style ${ }^{30}$ was used for qualitative data and was carried out with NVIVO V.10 software. ${ }^{31}$ The authors DPDBG and MCMBB independently (re) read the transcripts for an overall impression and identified patterns inside the categorisation scheme, which was composed of the factors from the implementation model. ${ }^{25}$ For each pattern, search terms were selected and a codebook was drafted through an iterative consensus process. After independent coding of several initial transcripts, the codebook was refined and used to guide the analysis of the remaining transcripts. Three reviewers (DPDBG, MCMBB and EM) independently applied the codebook and expanded it as the analysis proceeded. They continually met and reviewed findings. The authors YJLvEH and HJMV reviewed and discussed the findings. Since we aimed to cover a broad scope, data saturation (the ability to obtain additional new information) ${ }^{32}$ of the interviews was attained only in the final stage of the interview series.

\section{Ethics statement}

Ethical approval was given by the Maastricht University Medical Ethics Committee in July 2011. The study was considered an evaluation of daily practice and hence not subject to the Medical Research Involving Human Subjects Act. All participants were assured that data were handled confidentially and could not lead to their identification. Interviewees were also informed that they could terminate the interview at any time. Oral informed consent to use data collected for scientific research (including publication of the study findings) was obtained from every interviewee.

\section{Patient and public involvement}

Data were collected from patients regarding continuity of patient care, treatment success, patient compliance, perceived expertise, patient-centred care, safety, healthcare access, preferences and satisfaction. In doing so, patients were invited to fill out surveys and to participate in interviews. The findings from the perspective of patients will be published in a separate scientific paper, whereas all study findings, including those from patients, have been published in a Dutch study report that is publicly available at https://www.rijksoverheid.nl/ documenten/rapporten/2015/11/11/voor-bighouden. During a national conference about skill mix later this year, patient representatives will be invited to share their feedback on the study findings.

\section{RESULTS}

In accordance with the triangulation framework, quantitative and qualitative results are not presented separately. To distinguish between them, quantitative results are presented in regular font and qualitative results in italic font. In case of significant quantitative trends between values at $\mathrm{T} 0$ and $\mathrm{T} 2$, both values are presented in the text separated by an arrow ( $\mathrm{T} 0 \rightarrow \mathrm{T} 2)$, otherwise only values at T2 are presented. For each (qualitative) topic, the most supporting and appealing citation $(\mathrm{Qx})$ is presented in table 2.

The study flow is presented in figure 1. In total, 544/292/418 NPs, 186/244/355 PAs and 131/125/134 physicians filled in the questionnaires at T0/T1/T2; 9 NPs, 8 PAs, 16 physicians and 28 stakeholders were individually interviewed and 12 NPs, 16 PAs and 3 physicians attended the five focus group meetings.

\section{Implementation}

At T2, FPA was achieved for $83.5 \%$ of the NPs and $86.3 \%$ of the PAs (data not in table). Reasons for not achieving FPA included physician reluctance, lack of approval by management and/or in-process implementation. NPs and PAs went through several key steps to achieve FPA. These included making individual agreements with physicians, assembling working groups within the organisation, making (group) agreements with pharmacists, writing protocols, submitting protocols to management (Q1), arranging access to (digital) patient files, widely informing the organisation and training.

\section{Structures}

Background characteristics

Quantitative demographic data and work-related details of the participants are presented in 
Table 2 Quotes per topic

\begin{tabular}{lll}
\hline Number quote Topic & Quote \\
\hline Q1 & Implementation & $\begin{array}{l}\text { 'That is the next step, that for several departments those protocols have been prepared. } \\
\text { We are proposing this to divisional directors and psychiatrists, so they know how we } \\
\text { want to implement this and also to give them insight into our competence. This is so that } \\
\text { they are aware of how we work.' (NP) }\end{array}$ \\
Q2 & $\begin{array}{l}\text { 'We stated within our team of nurse practitioners, that within the institution one has to } \\
\text { collaborate with psychiatrists. That one does not just go at it alone. We have said: we } \\
\text { want to provide openness and draft protocols together.' (NP mental health) }\end{array}$
\end{tabular}

Q3 Positioning NP/PA

'Show us what the NPs/PAs can do! In fact, what do they add? What is the actual added value, how do they make this visible and how can we make sound agreements?' (stakeholder)

$\begin{array}{lll}\text { Q4 Resources } & \begin{array}{l}\text { 'If you don't allow NPs to open a DBC*, you won't know whether care will ultimately } \\ \text { become cheaper.' (stakeholder) }\end{array} \\ \text { Q5 } & \begin{array}{l}\text { Adherence with } \\ \text { protocols }\end{array} & \begin{array}{l}\text { 'Psychiatrists within the institution refuse to give NPs an opportunity to develop good } \\ \text { protocols, leaving them bound hand and foot.' (NP) }\end{array}\end{array}$

Q6 Consultation 'We talk with each other every day. Every two weeks there are formal consultations with the entire team. But in the workplace, there is daily consultation. During consultation hours, we are physically next to each other, you know. So, in this aspect, there are very short lines of communication.' (physician)

\begin{tabular}{|c|c|c|}
\hline Q7 & Competence & $\begin{array}{l}\text { 'When is someone competent? In the centre, we deal flexibly with this. This is } \\
\text { necessary when you have } 4 \text { NPs walking around. It also means that when they do ask } \\
\text { for consultation, it should run quite smoothly. If they really should prescribe drugs and } \\
\text { they believe themselves incompetent, they should consult us so we can give them the } \\
\text { background they need.' (physician) }\end{array}$ \\
\hline Q8 & Role & $\begin{array}{l}\text { 'What is NP's/PA's role within the medical team? I think, best to describe is being part of } \\
\text { it.' (physician) }\end{array}$ \\
\hline Q9 & Routine behaviour & $\begin{array}{l}\text { 'If it concerns nonstandard drugs, we will then write it down. The prescription must be } \\
\text { seen by neonatologist within } 24 \text { hours. When in doubt, or whatever else, I let someone } \\
\text { watch.' (NP) }\end{array}$ \\
\hline Q10 & Knowledge & $\begin{array}{l}\text { 'Pharmacotherapy is a skill in itself. Final responsibility sometimes covers knowledge of } \\
\text { additional medical subjects, of which they have no expertise.' (physician) }\end{array}$ \\
\hline Q11 & & $\begin{array}{l}\text { 'It is just that we need to ensure that all of the specific knowledge will be used. But it is } \\
\text { also reflected in the internship supervisor's assessments, of course I see them too, and } \\
\text { I notice that they all are satisfied with the quality of knowledge, as I personally am on a } \\
\text { day to day basis.' (physician) }\end{array}$ \\
\hline Q12 & & $\begin{array}{l}\text { 'What is not yet properly regulated is that we don't have a real budget for refresher } \\
\text { courses. It is extremely important for them to receive further training. As with the } \\
\text { specialist, who creates a budget through collective labour agreements. Unfortunately, } \\
\text { this is not yet possible for the PA. The interesting conferences and symposia are naturally } \\
\text { always abroad, which means that they have to travel abroad, but simply don't have the } \\
\text { money.' (PA) }\end{array}$ \\
\hline Q13 & Acceptance & $\begin{array}{l}\text { 'The advantages are increasingly being recognized by everyone. Furthermore, being } \\
\text { the constant of the outpatient clinical team, they take over several responsibilities. We } \\
\text { work there about one day every two weeks. They work there every day and therefore they } \\
\text { overhear more such as problems doctors' assistants face in their work. They recognize } \\
\text { them more quickly than we do, which means that problems can be resolved more rapidly. } \\
\text { Besides, because they work there every day and know a great deal, outpatient clinic staff } \\
\text { occasionally ask them what the standard approach is. There was support, but now this is } \\
\text { completely broad. No one has any doubts.' (physician) }\end{array}$ \\
\hline Q14 & & $\begin{array}{l}\text { 'This led to quite some issues with some people. Not so much a defined profession, } \\
\text { but the hierarchy that has arisen due to a long-time employment of certain colleagues. } \\
\text { People like healthcare psychologists cannot abide a nurse practitioner with a different } \\
\text { approach for the activities. Not so much a discipline, but personal views, I guess. Some } \\
\text { psychologists are very excited while others say 'what on earth are you doing.' (NP) }\end{array}$ \\
\hline Q15 & & $\begin{array}{l}\text { 'Of course, nurses like to work with more permanent people, which make it possible to } \\
\text { build up experience and expertise with each other.' (PA) }\end{array}$ \\
\hline
\end{tabular}


Table 2 Continued

\begin{tabular}{|c|c|c|}
\hline Number quote & Topic & Quote \\
\hline Q16 & & $\begin{array}{l}\text { 'How the organisation handles it, that is what I do not find entirely satisfactory. I must } \\
\text { say that I am the second NP in our hospital. How do they deal with the talks we have } \\
\text { held with the organisation? Do they respond and set up things more often in consultation } \\
\text { with you? It is a real pioneering role, you know, together with my colleagues. So yes, } \\
\text { organisational awareness is required. I must make things known, to set up by myself, to } \\
\text { organise by myself. The initiative comes from ourselves, rather than the organisation. It } \\
\text { is a pity. It is partly unfamiliarity, also with the role, and inadequately being informed or } \\
\text { involved. I think it comes from both sides, which is unfortunate.' (NP) }\end{array}$ \\
\hline Q17 & & $\begin{array}{l}\text { 'The NPs are very credible care providers for patients, if patients have confidence in NPs } \\
\text { knowledge, which is certainly present, and NPs demonstrate they know their boundaries. } \\
\text { Nurse's accessibility and communication make them a credible care provider for patients. } \\
\text { Naturally, from time to time patients should be seen by a physician. But very often they } \\
\text { return to the NP, as soon as possible.' (physician) }\end{array}$ \\
\hline Q19 & & $\begin{array}{l}\text { 'What makes it very difficult is that the insurers within the psychiatry, the mental } \\
\text { healthcare, currently indicate that they do not recognise NPs as primary practitioners. } \\
\text { They extremely prohibit us from treating.' (NP) }\end{array}$ \\
\hline Q20 & Responsibilities & $\begin{array}{l}\text { 'Yes, this is more of a learning process. As it is getting better and I can agree with their } \\
\text { proposals, I increasingly let them go. Not before, regardless any amendment. Sure, that } \\
\text { amendment makes it easier, but it must be justified.' (physician) }\end{array}$ \\
\hline Q21 & Legislation & $\begin{array}{l}\text { 'The letters of the law do not allow me to order chest photos, CT-scans or anything with } \\
\text { radiation. This is hard to grasp in reserved procedures. Because that is also related to } \\
\text { radiation, captured in another law. PA's daily work is hampered by the absence of a } \\
\text { reserved procedure on ordering radiation sources.' (PA) }\end{array}$ \\
\hline
\end{tabular}

${ }^{*} \mathrm{DBC}$ (in Dutch Diagnose Behandeling Combinatie) is a hospital funding model based on fixed prices for a combination of diagnosis and treatment.

NP, nurse practitioner; PA, physician assistant.

the online supplementary table 1 . The results showed no statistically significant differences over time for age, gender, prior education or NP specialisation.

\section{Collaboration/Collaboration}

The number of physicians with whom NPs and PAs collaborated did not change after the introduction of FPA (data not in table). Over half of the PAs (T2: 55.9\%) worked in collaboration with $>6$ physicians and about one-third (T2: $32.4 \%$ ) in collaboration with three to six physicians. NPs worked in slightly smaller teams: equal proportions worked in a collaboration with three to six physicians (T2: $37.4 \%$ ) and $>6$ physicians (T2: $37.4 \%$ ). A small percentage of NPs (T2: 1.7\%; those who worked in an ambulance setting) did not collaborate with any physicians. All NPS/ PAs and physicians emphasised the importance of collaboration with each other (Q2).
Positioning NP/PA

The positioning and visibility of the NP/PA was associated with the implementation of FPA, but FPA was also used to achieve better positioning. Higher management and external stakeholders asked for evidence of the added value provided by NPs/PAs (Q3).

\section{Resources}

Funding of NPs/PAs varied. They were employed by the organisation, but also by associations of physicians. Registration of their operations was not transparent, although the necessity was recognised by the directorate. Clear registration was considered indispensable to establish the cost-effectiveness of using NPS/PAs (Q4).

\section{Processes}

Adherence with protocols/Adherence with protocols

Almost every interviewed NP/PA worked according to (national) guidelines and under clear work instructions. Cooperation 
agreements with physicians as part of the protocols were occasionally drafted. Written protocols were still not present in all settings (online supplementary table 2, T2: NPs: $15.2 \%$, PAs: $23.7 \%$ ). If present, they often failed to be completely satisfactory (T2: NP 44.4\%, PA 36.2\%). Deficiencies mainly concerned the distribution of tasks (T2: NP 21.9\%, PA $14.7 \%$ ) and responsibilities (T2: NP 22.6\%, PA 10.6\%). This was not due to ambiguities, but to lack of formally recording the mutual agreements. About half of the NPs/PAs indicated that protocol development was still in process; but some stated that the process was restrained (Q5) since physicians wanted to maintain overall responsibility. For about half of the NPs and a third of the PAs the protocols were personalised and recorded (online supplementary table 2, T2: NP 57.4\%, PA 36.7\%). Of those with personalised protocols, less than half (T2: NP $43.0 \%$, PA $38.5 \%$ ) were checked for adherence.

\section{Consultation/Consultation}

Availability of physicians for consultation, if needed by the NP/PA, was considered good (online supplementary table 3, T2: NP 63.3\%, PA 79.2\%) and thus NPs/PAs were satisfied (T2: NP 91.6\%, PA 95.0\%). The frequency of the consultation varied. Two trends between $\mathrm{T} 0 \rightarrow \mathrm{T} 2$ were statistically significant: more NPs/PAs consulted physicians more than once a day (NP: $11.9 \% \rightarrow 25.1 \%$, PA: $29.2 \% \rightarrow 52.0 \%)$ and fewer NPs only consulted them once a day $(33.3 \% \rightarrow 18.1 \%)$. One-third of the NPs who consulted physicians monthly worked in an acute setting. The consultation was mainly face-to-face or by phone. Ad hoc consultation was always possible, but structural consultations about less acute issues were hard to schedule and in-depth conversations were not always possible. Most NPs/PAs had structural work meetings, the frequency of which were highly dependent on the setting (Q6). In hospitals, NPs/PAs participated in multidisciplinary meetings, which physicians sometimes considered as a threat.

\section{Competence/Competence}

Significantly more NPs and PAs replied affirmatively to most response categories with methods to assess their competence for reserved procedures. At T2 compared with T0 (online supplementary table 4): more NPs and PAs assessed their competence, above all, based on patient characteristics (NP $27.0 \% \rightarrow 68.8 \%$, PA $18.4 \% \rightarrow 65.3 \%$ ) next to the specific procedure (NP $26.0 \% \rightarrow 27.1 \%$, PA $23.0 \% \rightarrow 44.6 \%$ ) and the education/training (NP $13.0 \% \rightarrow 28.8 \%$, PA $9.3 \% \rightarrow 35.6 \%$ ). An increase was observed in tendency for physicians to issue certificates of competence and/or endorsements (NP $11.0 \% \rightarrow 26.5 \%$, PA $2.3 \% \rightarrow 27.7 \%$ ). Small percentages of NPs/PAs left the assessment of their competence to the responsibility of the physicians (T2: NP 7.7\%, PA 6.9\%). Physicians usually assessed this competence based on their training (T2: physician NP 34.3\%, physician PA 26.9\%) or left the assessment to the NP/PA themselves (T2: physician NP 33.3\%, physician PA $28.8 \%$ ). Significantly less physicians assessed the competence of NPs based on the specific procedure (physician NP $47.5 \% \rightarrow 23.0 \%$ ). Interviews showed that physicians had a growing confidence in NPs/PAs to guard their own boundaries (Q7). Before the amendment, half of the NPs reported that the physicians checked their prescriptions, whereas after the amendment this went down to just a quarter (data not in table; $47.1 \% \rightarrow 22.5 \%$ ). For PAs, there was no shift: before and after the amendment, a quarter of the prescriptions were checked (T2: 24.7\%). The percentages of NPs/PAs asking for physician consent afterwards decreased significantly (NP $98.2 \% \rightarrow 29.1 \%$, PA $52.9 \% \rightarrow 30.6 \%)$.

\section{Role}

All NPs/PAs had an integrated role in the treatment team (Q8). Following the amendment, the role of some NPs/PAs had changed to be more autonomous and in many cases they became seen as role models.

\section{Routine behaviour}

Some reserved procedures could not be performed routinely. To maintain expertise some NPS/PAs regularly worked in a different setting. When prescribing non-standard medication or medication for patients with multimorbidity, most NPs/PAs consulted a physician or had the prescription checked afterwards (Q9).

\section{Knowledge/skills}

Most NPs and PAs judged their knowledge to independently prescribe as sufficient (data not in table; T2: NP 79.9\%, PA 76.1\%), which did not change significantly over time. NPS/PAs reported that because of the limited prescription formulary, their knowledge was good enough. Alike, physicians stated that NPs/PAs had an adequate level of pharmacotherapy knowledge, however a small proportion disagreed (T2: physician NP 9.1\%, physician PA 14.5\%) and explained that sometimes broader knowledge of other medical specialisations was essential (Q10).

Interviews showed that NPS/PAs and physicians agreed that $N P / P A$ knowledge was the most important requirement for FPA, which was generally perceived as adequate (Q11). To maintain knowledge, ongoing training occurred in different ways: internal training (strongly setting dependent), visiting (inter)national conventions/symposia and attending courses. Lack of adequate funding was often a limiting factor (Q12) and caused problems with obtaining accreditation points. The restricted accredited training services also hindered the gathering of these points. However, most NPs/PAs indicated they were ultimately able to submit a sufficient amount of accreditation points to the National Accreditation Registry governing their profession (data not in table; T2: NP 85.7\%, PA 78.6\%).

$\mathrm{NP} / \mathrm{PA}$ skills to perform reserved procedures were rated as excellent (data not in table; T2: NP $83.6 \%$, PA 90.7\%, physician NP 78.5\%; physician PA $87.8 \%$ ).

\section{Acceptance}

The acceptance of NPS/PAs could be divided into:

Closely collaborating physicians: Interviewed NPs/PAs were well accepted by those physicians. The introduction of the $N P / P A$ was most often initiated by a single physician and after initial scepticism the role was widely supported by as- 
sociated physicians. Good positioning was considered to be facilitating (Q13).

Other physicians: The acceptance by other physicians var$i e d$. The more ignorant physicians were of NP/PA role, the weaker their support. NPS/PAs talked about the process of gaining confidence as slow going and there remained scepticism among physicians. The level of support for prescription authority was strongly dependent of the type of medication (Q14).

Nurses: Nurses supported NPs/PAs for being a consistent and (often) having a nursing background (Q15).

Management: The lowest management levels widely accepted NPS/PAs. The extent of support from the highest levels was strongly dependent on the setting being 'NP/PA-minded', which led to differences in policy, guidelines and agreements (Q16).

Patients: Physicians and NPs/PAs pointed out that patients strongly accepted NPs/PAs for their accessibility and continuity. Most patients could not tell the difference between NPS/ PAs and physicians (Q17).

Pharmacists: Pharmacists contributed to the implementation of FPA when quality was guaranteed and clear agreements were made. The provision of a formal approval of a physician added value (Q18).

Health insurance companies: In mental healthcare the proper functioning of NPs was hindered by their lack of recognition as primary caregivers, which subsequently caused invoice problems (Q19).

\section{Outcomes}

\section{Appropriate performance}

Most NPs, PAs and physicians agreed that implementation of FPA led to improvement in healthcare for particular groups of patients (data not in table; T2: NP $88.7 \%$, PA $84.6 \%$; physician NP $66.6 \%$, physician PA $61.2 \%$ ). When asked for clarification, they said NPs and PAs provided continuity and could work on quality improvement, previously the responsibility of rotating trainee doctors.

The proportion of NPs/PAs performing (indicating, executing, delegating) a reserved procedure (quick scan, table 3) increased during the measurement period $\mathrm{T} 0 \rightarrow \mathrm{T} 2$ for all procedures (except for cardioversion and endoscopy by PAs). Increases were statistically significant for catheterisations performed by PAs $(25.3 \% \rightarrow 26.7 \%)$, prescriptions by NPs and PAs (NP $55.2 \% \rightarrow 72.5 \%$, PA $57.9 \% \rightarrow 81.6 \%)$ and small surgical procedures by NPs and PAs (NP 22.3\% $\rightarrow 31.0 \%$, PA $37.9 \% \rightarrow 52.9 \%)$. Trends for mean $(95 \% \mathrm{CI})$ number of monthly performed procedures were less coherent. There were significant increases in PA injections (19.8 $(13.3-26.2) \rightarrow 37.2 \quad(27.5-47.6))$, punctures (9.5 (5.7$13.3) \rightarrow 19.6(12.5-26.8))$ and small surgical procedures $(15.5(9.3-20.9) \rightarrow 30.0(22.8-38.3))$.

Compared with T0, NPs/PAs performed all procedures at T2 more autonomously and without consulting a physician.
The proportion of procedures performed on authorisation of NPs significantly increased for prescribing $(65.7 \% \rightarrow 74.5 \%)$ and small surgical procedures $(62.7 \% \rightarrow 88.7 \%)$. As for prescribing, no differences in authorisation mode between new and repeat prescriptions emerged.

The proportion of procedures performed on authorisation of NPs/PAs without consultation with a physician significantly also increased for prescribing (NP $27.3 \% \rightarrow 51.0 \%$, PA $35.8 \% \rightarrow 45.6 \%)$ and small surgical procedures performed by NPs $(40.0 \% \rightarrow 68.1 \%)$. At the same time, the proportion of all procedures delegated to another care provider significantly increased (NP $1.7 \% \rightarrow 13.0 \%$, PA $0.1 \% \rightarrow 12.4 \%)$.

The mean $(95 \% \mathrm{CI})$ contact between physicians and $\mathrm{NPs} / \mathrm{PAs}$ regarding procedures (from the perspective of the physician) significantly decreased for puncture (NP $26.9(14.9-38.9) \rightarrow 6.9(2.2-11.5)$, PA $13.9(9.6-18.1) \rightarrow 6.3$ $(3.7-8.9))$ and for all procedures performed by PAs $(107.2(82.9-131.5) \rightarrow 53.9(38.6-69.3))$. Average contact also decreased for all other procedures (except injection), however none met statistical significance.

For all procedures, the contact between physicians and NPs changed over time. Significantly less contact was made to assign procedures to the NP $(64.8 \% \rightarrow 41.5 \%)$ and more contact was made for consultations requested by the NP. The proportion of assignments did not change for PAs. Furthermore, the proportion of assignments to NPs significantly decreased for all specific procedures, with the exception of catheterisation.

From the perspective of physicians, the overall mean (95\% CI) assignment time significantly increased (physician NP $3.8(1.0-5.0) \rightarrow 5.9(1.6-10.0) \mathrm{min}$; physician PA $3.5(0.5-5.0) \rightarrow 6.6(2.0-10.0) \mathrm{min})$. Increases were also observed for all separate procedures except for prescribing performed by NPs.

Above measures could not be determined for cardioversion/defibrillation or endoscopy due to a lack of data.

\section{Responsibilities}

A few NPS/PAs reported that the amendment was not a tipping point for the way they performed a reserved procedure. For most $N P s / P A s$, autonomy increased for the range of reserved procedures they were allowed to (independently) perform and they were less rigorously checked. A learning curve was mentioned often (Q20).

\section{Efficiency}

The mean duration (95\% CI) of all procedures in minutes (table 3), including consultation or assignment time, significantly decreased over the time points (NP 9.9 (9.3$10.5) \rightarrow 8.6 \quad(8.2-9.0)$, PA $8.8 \quad(8.1-9.4) \rightarrow 7.4 \quad(6.9-7.8))$, just like some of the specific procedures, namely catheterisation (NP 13.4 (11.9-15.0) $\rightarrow 9.1$ (8.1-10.2), PA 10.3 $(8.9-11.7) \rightarrow 7.2(6.2-8.2))$ and injections performed by PAs $(7.3(6.4-8.3) \rightarrow 5.6(4.7-6.4))$.

Nearly every NP/PA and physician opined, during all measurements, that healthcare for certain groups of 


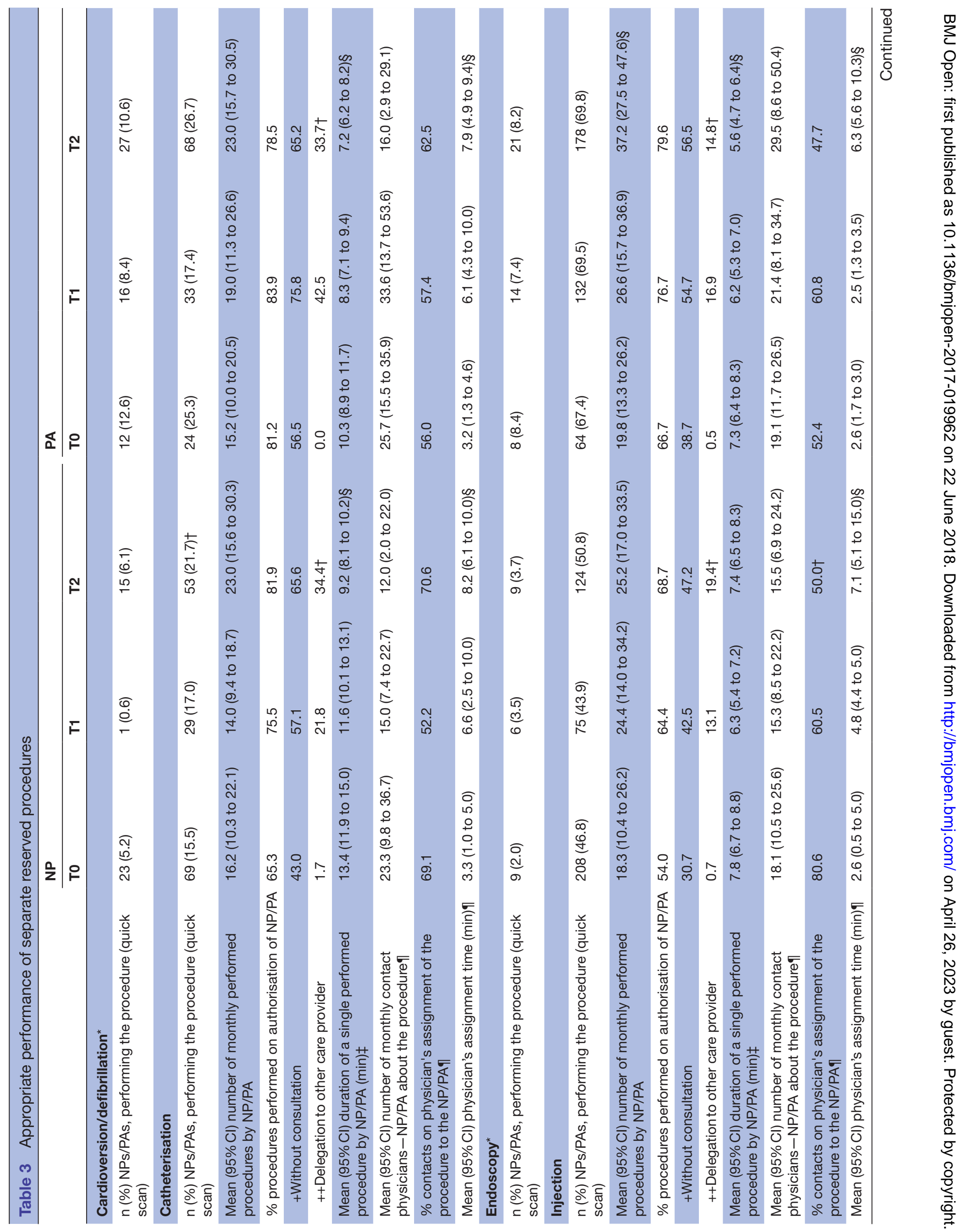




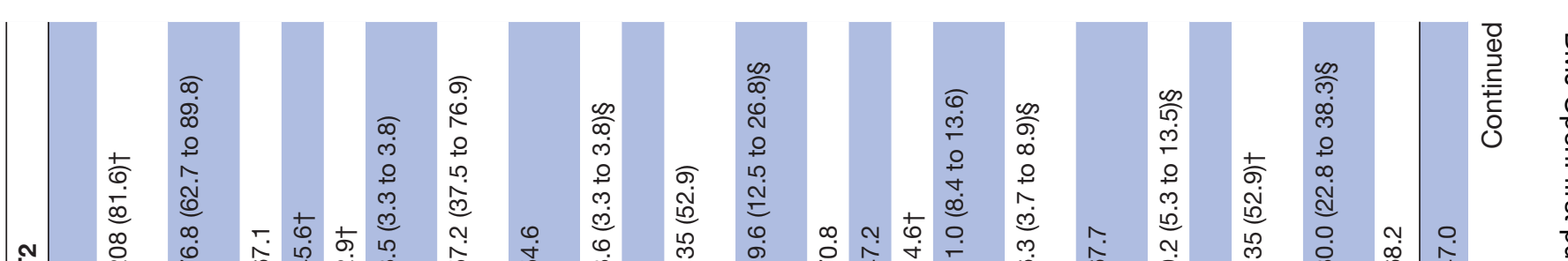

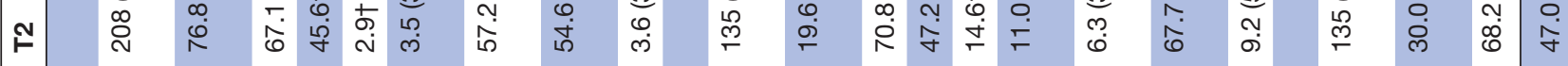

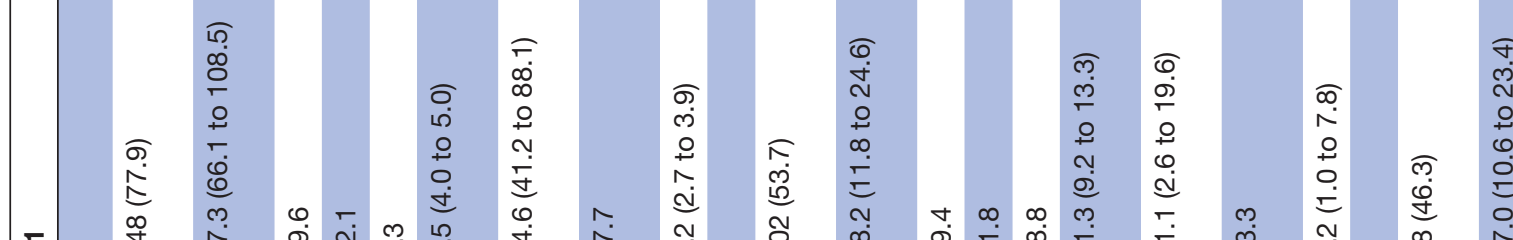

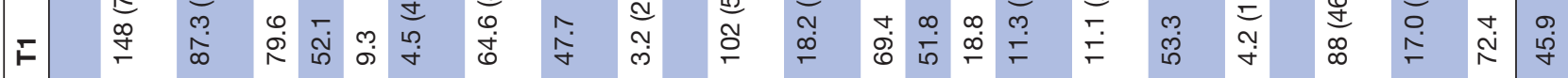

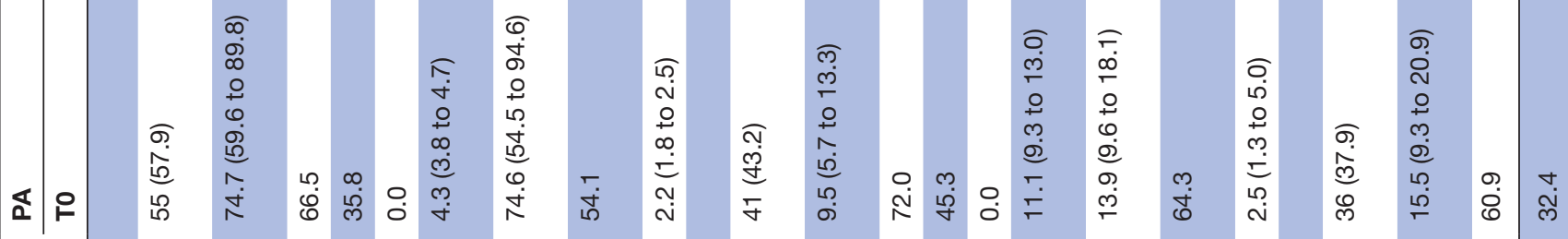

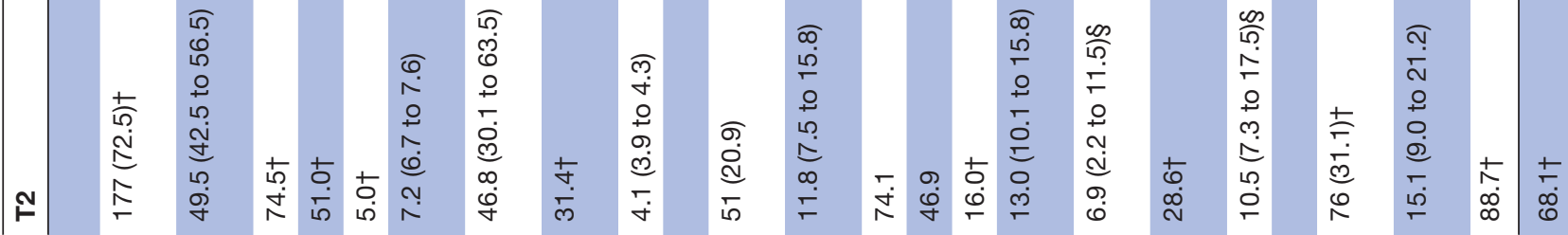

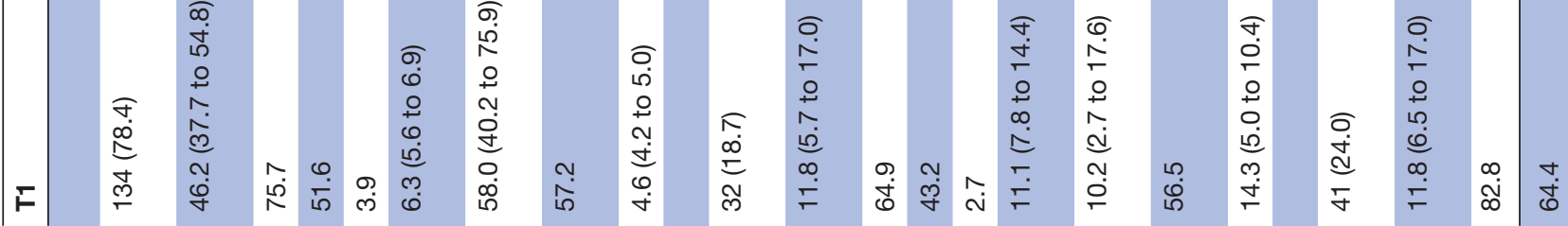

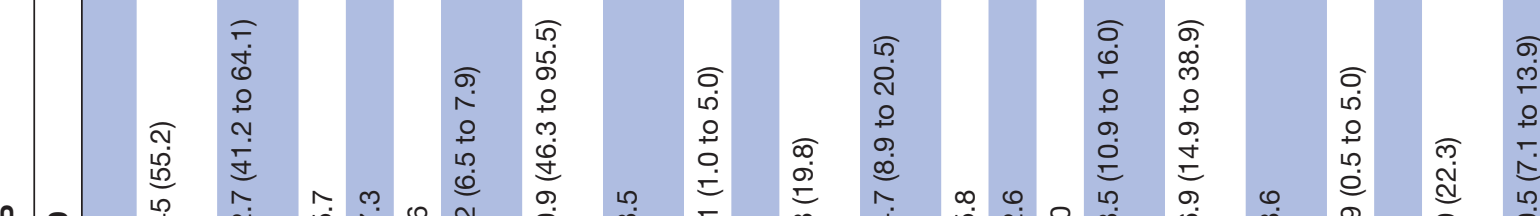

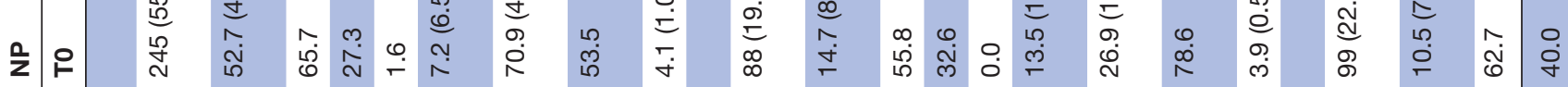

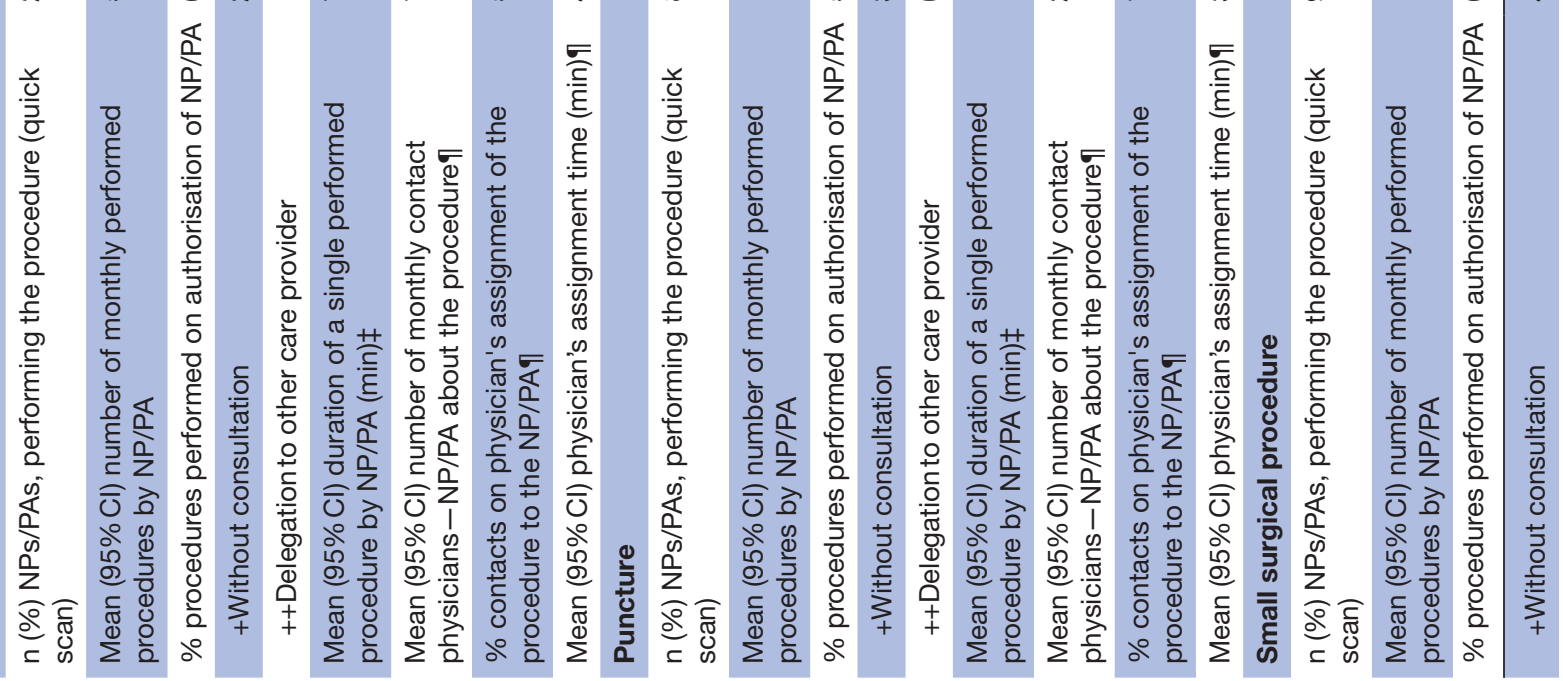

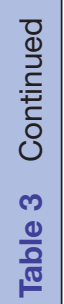
일 응

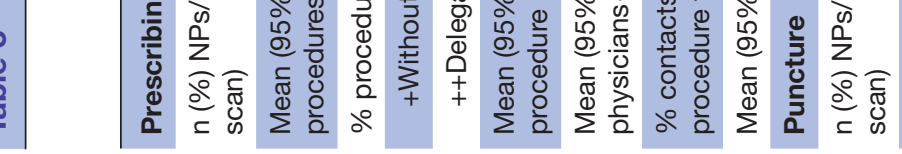




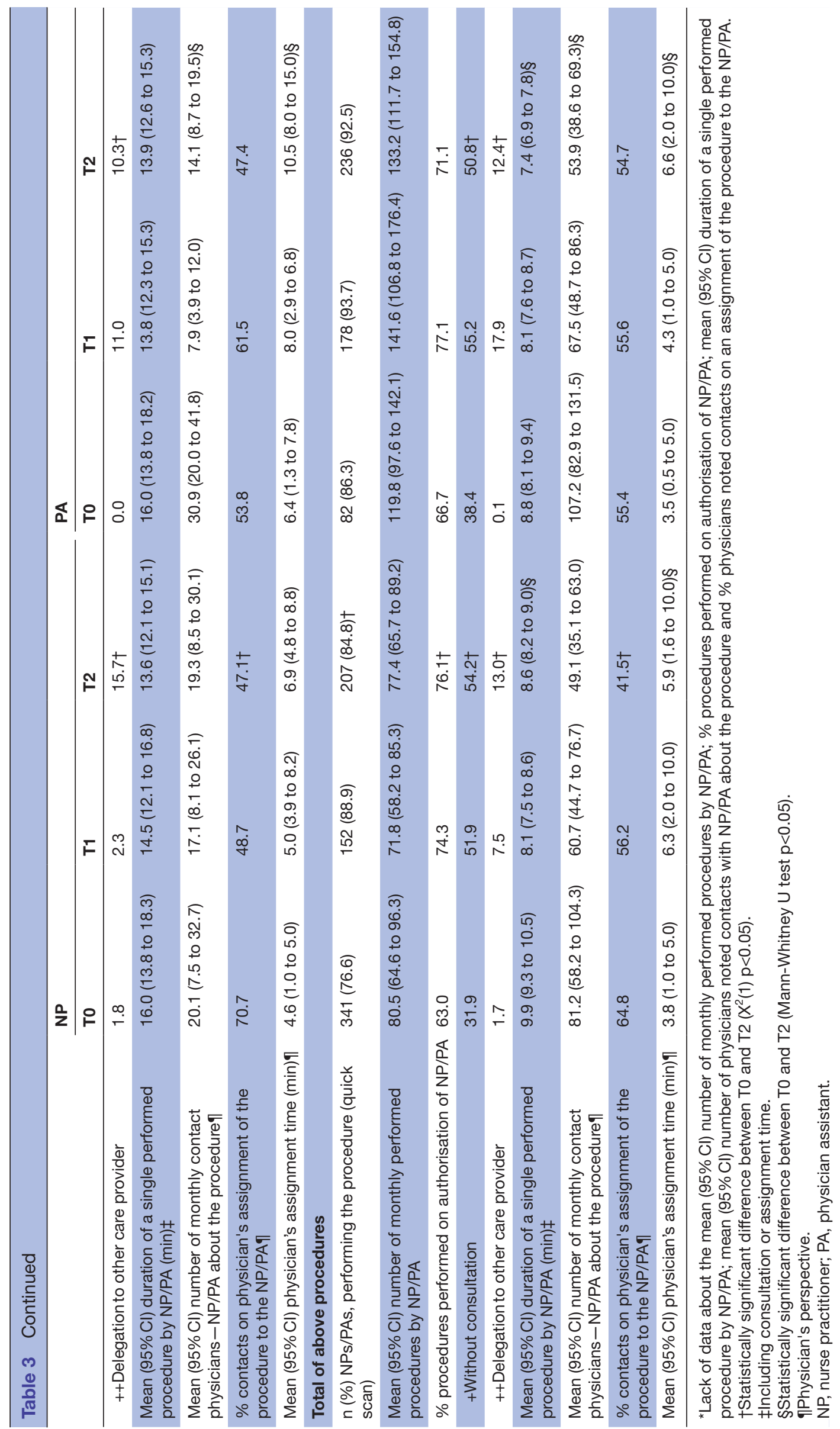


patients became more efficient after granting FPA for specific reserved procedures (data not in table, T2: NP $96.4 \%$, PA $94.8 \%$, physician NP $80.4 \%$; physician PA $83.9 \%)$.

In answer to the question whether physicians noticed a change in contact with patients following the introduction of NPs/PAs, only a minority controverted (online supplementary table 5, T2: physician NP 23.9\%, physician PA $13.5 \%)$. Half of the physicians collaborating with an NP $(56.8 \%)$ and a quarter of the physicians collaborating with a PA $(28.8 \%)$ saw patients with more complex medical needs. Moreover, a quarter of physicians reported they saw less patients (physician NP 26.1\%, physician PA $26.9 \%$ ) and another quarter (those collaborating with a PA) also reported they performed less medical procedures $(25.0 \%)$. About one in six physicians reported that patient contact lasted longer.

\section{Legislation}

The amended law was persistently considered to be an improvement over previous regulation (data not in table; T2: NP 94.0\%, PA 95.7\%, physician NP 78.8\%, physician PA 70.9\%) and in line with daily practice (NP T2: $79.9 \%$; PA T2: $72.2 \%$; physician NP T2: $83.8 \%$; physician PA T2: $70.8 \%)$. Yet, a reasonable percentage of care providers indicated that the list of reserved procedures did not go far enough, though this did significantly decrease over time (NP $17.7 \% \rightarrow 9.5 \%$; PA $31.8 \% \rightarrow 21.6 \%$; physician NP $29.7 \% \rightarrow 7.2 \%$; physician PA $37.3 \% \rightarrow 15.2 \%$ ). Specific procedures mentioned to be lacking included ascites tapping, determination of death and performing/ordering radiotherapy (Q21). It was also said that the described list should be regularly re-evaluated as to whether it corresponds to daily practice (Q22).

\section{DISCUSSION}

In Dutch health reform policy, task shifting has been prioritised in the form of granting FPA to NPs and PAs, specifically for the performance of cardioversion/defibrillation, catheterisation, endoscopy, injections, prescribing, puncture and small surgical procedures. FPA's introduction was accompanied by a nationwide evaluation of its effects on the processes and outcomes of care for purposes of further decision-making. The present article includes a basic section of the evaluation study.

FPA's legalisation was driven by the need of the professional field. ${ }^{33}{ }^{34}$ In our study, this need was clearly reflected in the initial presence of informal practice and further exploitation of the legal possibilities. Prior to the introduction of FPA, most NPs/PAs frequently performed reserved procedures on their own authority. After the introduction, the proportion of NPs/PAs performing reserved procedures increased, as did autonomous performance.

The implementation of FPA was found to be strongly dependent on the setting, as was already shown for prescriptive authority. ${ }^{35}$ Scepticism of physicians and medical boards hampered the full implementation, especially in areas of mental health, which was demonstrated prior to the introduction of FPA. ${ }^{36}$ This barrier has also emerged in other countries where restrictions that limit a more fully practising authority were addressed. $^{734}$ 37-41 The main objection to FPA is also reflected in the present study: following an extensive clinical education, physicians are better trained at managing patients with complex health problems. ${ }^{142}$

Three-quarters of the studied reserved procedures were performed on the NP's/PA's own authority. For about a quarter of those procedures, consultation with a physician was needed. The observed need for consultation might have been due to a sense of uncertainty, emphasising the necessity of a collaborative relationship with a physician. Consultation might also have been necessary when additional rules and limitations had been imposed. ${ }^{35}$ Indeed, there was a growing trend to set out specified conditions (eg, type of procedures, patient groups to treat independently) in protocols. Restriction of NP activities to a narrower scope of practice than legally authorised has been seen in other studies. ${ }^{43-45}$

The mean number of contacts between physicians and NPs/PAs about a procedure decreased over time with FPA, as did the overall procedural time. However, the mean physician's assignment time increased. A plausible explanation for this might have been the shift in the physician's patient population towards fewer patients that had more complex health problems. Assigning a reserved procedure to an $\mathrm{NP} / \mathrm{PA}$ subsequently required more instruction. Also, NPs/PAs treated most of their patients independently. Physicians were only consulted when the NP/PA was in doubt, which also resulted in longer consultation time. Literature has confirmed the existence of such a patient shift, where physicians tend to have older patients with a greater number of comorbidities or severity of illness. ${ }^{46}$ However, more research on this patient shifting hypothesis is needed. Furthermore, to test the hypothesis around cost-effectiveness of FPA, more and broader research on (additional) operations (eg, ordering diagnostic imaging and pathology, and prescribing patterns) is needed because the effect of FPA on total healthcare spending is inconclusive.

A first proxy to efficient granting of FPA can be found in the mean duration for NPs/PAs to perform a procedure. This duration decreased due to elimination of dispensable assignment time and, to a lesser extent, consultation time with physicians. One of the presumed benefits of FPA is removing delays in care when a physician's authorisation is needed prior to initiation of medications or diagnostic testing. ${ }^{47}$ Saving time for both NPs/ PAs and physicians may result in more time for patient care, thereby improving access to care. ${ }^{42}$ To our knowledge, the present study is the first to account for time in this regard.

As access to care improves, utilisation will run in parallel. Although prices could decrease for NP/PA services, the number of services provided may increase, raising overall costs of healthcare. ${ }^{42} 48$ For example, USA granting NPs 
independent prescriptive authority had higher rates of prescriptions filled and higher prescription costs, leading to higher overall costs. ${ }^{46}$ At the time of this evaluation NP's/PA's operations were registered in the name of the supervising physician or department instead of the individual NP/PA. This has since been adjusted. ${ }^{49-51}$

Legal cross-compliance requirements were mainly fulfilled. The most important requirement was the NP's/PA's competency. NPs/PAs were very conscious of the boundaries of their competency, usually based on the actual situation, and on the individual patient associated with the specific reserved procedure. After the introduction of FPA, physicians left the assessment of NP's/PA's competency more and more to the NPs/PAs themselves. Physicians began to provide slightly more certificates of competence to, and regular assessment for, NPs/PAs. An insufficient budget for training sometimes obstructed maintenance of knowledge and therefore competency. Another cross-compliance requirement included the consultation structure for the performance of reserved procedures. NPs/PAs were very satisfied with the consultation structure and physician's availability for consultation. Lastly, NPs/PAs performed reserved procedures according to (national) guidelines and extracted (and personalised) protocols. The protocols sometimes showed deficiencies in recording local cooperation agreements, like the distribution of tasks and responsibilities. According to the study protocol, ${ }^{14}$ workload and the perspective of patients (fourth questionnaire): continuity of patient care, treatment success, patient compliance, perceived expertise, patient-centred care, safety and healthcare access, in addition to patient preference and satisfaction in the patient interviews, were the subjects of evaluation. These are elaborated on elsewhere due to the extensiveness of results.

Despite their ability to provide diverse health services independently, NPs worldwide are constrained from doing so because of restrictive state laws and regulations. ${ }^{383952-57}$ Little is known about PA regulations and authorities. The legal status of PAs is often not resolved, resulting in their limited authority. ${ }^{57}$ The common denominator seems to be that PAs, as physician extenders, work under supervision of physicians and rarely practise (or prescribe) independently. ${ }^{53}{ }^{57-61}$ As a result, there is a great lack of studies measuring the effectiveness of fully independent practices, as also seen above. This is reinforced by the fact that research isolating the effects of NP/PA from a whole healthcare chain on various outcomes is limited. Most studies on the role of NP/PA address quality of care and there is a growing body of evidence, based on systematic reviews, that NPs provide care at least equivalent to that of physicians in terms of health status, satisfaction, treatment adherence, patient risk and use of specialists. ${ }^{262-66}$ However, systematic reviews do not distinguish between degrees of independence, nor any changes thereto over time. For PAs, no conclusive scientific evidence is available at all. Nevertheless, internationally there are some indications that easing scope-of-practice regulations can increase quality of care. Traczynski and Udalova, ${ }^{67}$ in a study on healthcare utilisation and health outcomes, concluded that US states without restrictive NP regulations scored better on patient-reported available time, listening to concerns and understandable explanations. Furthermore, they saw an increase in annual check-ups and a long-term reduction in avoidable emergency room visits after gaining NP independence. Similar studies within the Dutch setting are advisable.

The independent performance of medical procedures by NPs and PAs as part of practice authority is rarely specified in regulations. In literature, various illustrations of, to a greater or lesser extent, independent performance of medical procedures can be found in different settings in Australia, England and USA. These procedures include abdominal drainage, biopsy, bronchoscopy, cardioversion, chest tube insertion or removal, endoscopy, fineneedle aspiration, placement of (pulmonary) arterial or (peripherally) central catheter, placement of extraventricular drain, intravitreal/joint/carpal tunnel injection, lumbar puncture, paracentesis, sedation/anaesthesia, thoracentesis, removal of intracranial pressure monitor and thoracostomy. ${ }^{58}{ }^{61}{ }^{68-78}$ Here too, more research is needed.

\section{Strengths and weaknesses of the study}

A key strength of this study is the mixed-methods and triangulation approach. The introduction of FPA, a complex and delicate intervention, asked for an evaluation from multiple perspectives in a broad range of areas. Quantitative data alone might not cover the full picture, as it neither illuminated how or why FPA was implemented (or not), nor enabled contextualising. Multifaceted triangulation was used to minimise bias. We applied (mixed) methods triangulation to reduce single method bias, and data triangulation through various data sources, including time (three time points), participant (NPs/ PAs, physicians, patients, stakeholders) and site (organisational setting, geographical coverage). Investigator triangulation, combining multiple interviewers and data analysts, supported by a solid interview guide and codebook, and peer review of the findings, was used along with theory triangulation (two theoretical frameworks) to further reduce bias. This approach empowered us to overcome moderate survey participation and provided a reliable and representative picture of the effectiveness and efficiency of FPA for NPs/PAs.

Another strength of this study is its potential for crosscountry comparison. The frameworks used for both quantitative and qualitative data are internationally accepted and applied. Besides, the study design captures the impact of the regulation on generic outcomes and the underlying mechanism and their association.

There are, nonetheless, some limitations of this research which require consideration. First, only a small proportion (12\%) of the NPs/PAs, and hence also physicians, completed the surveys at all time points. Observed differences between $\mathrm{T} 0$ and $\mathrm{T} 2$ in those population-level 
results could be attributed to differences in group composition. However, longitudinal subanalysis of the individuals completing all surveys showed equal (ie, not statistically significant due to insufficient statistical power) trends. Moreover, these trends were confirmed by qualitative data. Second, according to the traditional positivist/empiricist approach of demonstrating causality, the used One Group Pre-post test design has its shortcomings. Over the past decade, new more advanced causality models were developed, all reflecting three key criteria: the cause precedes the effect, there is a certain relationship between cause and effect, and other plausible alternatives are eliminated. ${ }^{79}$ With the mixed methods design we approached all criteria. The quantitative methods were used to establish a relationship between the cause and effect, before the amendment and thereafter. Qualitative data were used to describe the processes after the amendment and to eliminate other plausible alternatives. A third limitation of this study is related to external validity. The survey sample of collaborating physicians did not reflect the total population of physicians. It was considered important to include only those physicians who had experience in working with NPs/PAs. However, formal points of view of professional organisations, representing all physicians, were expressed in expert interviews. Finally, the novel survey tool was not tested for reliability and validity. However, the survey tool was based on validated instruments and there were no indications for issues regarding reliability and validity.

\section{Conclusion and policy implication}

The study showed that for some NPs/PAs informal practice was legalised by the law amendment enabling FPA, whereas others were encouraged to further develop their role. New opportunities for NPs/PAs to independently indicate, perform and delegate catheterisations, injections, prescriptions, punctures and small surgical procedures were highly exploited. Notwithstanding, for elective cardioversion/defibrillation and endoscopy, lack of data did not allow for any conclusions. Care processes were organised more efficiently, and care was provided by the most appropriate healthcare provider. These study findings support the policy initiative to improve the effectiveness of care delivery by granting FPA to NPs and PAs. Hence, our advice is to turn this temporary policy initiative into a definite one.

Acknowledgements We are grateful to Eva Mulkers for her assistance with NVIV0 and Kristine Stickney for her editorial work.

Contributors DPDBG, MCMBB, YJLvEH and HJMV significantly participated in the conception and design of the study and the interpretation of the data. DPDBG and MCMBB conducted the interviews, collected the data and were responsible for the statistical analyses. BABE provided input for the cost study. DPDBG wrote the first and subsequent drafts of the paper, and all (DPDBG, MCMBB, YJLvEH, BABE, CDD and HJMV) contributed to editing and refining. All authors read and approved the final manuscript.

Funding This work was supported by the Dutch Ministry of Health, Welfare and Sport (March 2011 grant number 320434) and endorsed by the professional organisations Nurses and Carers Netherlands department for nurse practitioners (NCN NP, in Dutch V\&VN VS), National Association of Physician Assistants (NAPA) and the Royal Dutch Medical Association (RDMA, in Dutch KNMG). The report of this study was presented to the Dutch Minister and subsequently to the House of Representatives for further decision-making. The report, in Dutch, is available at: https://www.rijksoverheid.nl/documenten/rapporten/2015/11/11/voor-bighouden.

Competing interests None declared.

Patient consent Not required.

Ethics approval Ethical approval for this study was obtained from the Maastricht University Medical Ethics Committee in July 2011 (reference number METC 11-5-045).

Provenance and peer review Not commissioned; externally peer reviewed.

Data sharing statement The data sets generated during and/or analysed during the current study are available from the corresponding author on reasonable request.

Open access This is an open access article distributed in accordance with the Creative Commons Attribution Non Commercial (CC BY-NC 4.0) license, which permits others to distribute, remix, adapt, build upon this work non-commercially, and license their derivative works on different terms, provided the original work is properly cited and the use is non-commercial. See: http://creativecommons.org/ licenses/by-nc/4.0/

(c) Article author(s) (or their employer(s) unless otherwise stated in the text of the article) 2018. All rights reserved. No commercial use is permitted unless otherwise expressly granted.

\section{REFERENCES}

1. Dennis S, May J, Perkins D, et al. What evidence is there to support skill mix changes between GPs, pharmacists and practice nurses in the care of elderly people living in the community? Aust New Zealand Health Policy 2009;6:23.

2. Laurant M, Reeves D, Hermens R, et al. Substitution of doctors by nurses in primary care. Cochrane Database Syst Rev 2005;2:CD001271.

3. Weeks G, George J, Maclure K, et al. Non-medical prescribing versus medical prescribing for acute and chronic disease management in primary and secondary care. Cochrane Database Syst Rev 2016;107:Cd011227.

4. Moote M, Krsek C, Kleinpell R, et al. Physician assistant and nurse practitioner utilization in academic medical centers. Am J Med Qual 2011:26:452-60.

5. Pulcini J, Jelic M, Gul R, et al. An international survey on advanced practice nursing education, practice, and regulation. $J$ Nurs Scholarsh 2010;42:31-9.

6. Xue Y, Ye Z, Brewer C, et al. Impact of state nurse practitioner scopeof-practice regulation on health care delivery: Systematic review. Nurs Outlook 2016;64:71-85.

7. Delamaire M, Lafortune G. Nurses in Advanced Roles: A Description and Evaluation of Eperiences in 12 Developed Countries. OECD Publishing: OECD Health Working Papers, 2010.

8. Maier CB. The role of governance in implementing task-shifting from physicians to nurses in advanced roles in Europe, U.S., Canada, New Zealand and Australia. Health Policy 2015;119:1627-35.

9. (NVDV) NVvDeV. Leidraad Laser en flitslamp. Utrecht, 2016.

10. Ministerie van Volksgezondheid WeS. WeS MvanV, ed. Kamerbrief betreffende Beleidsreactie rapport RIVM: 'Laseren en aanverwante behandelingen als voorbehouden handeling in de Wet BIG', 885694145417-MEVA: Den Haag, 2016.

11. Bruijn De-Geraets D, Bessems-Beks M, Eijk van-Hustings $Y$, et al. voorBIGhouden. Eindrapportage Evaluatieonderzoek Art. 36a Wet $B I G$ met betrekking tot de inzet van de Verpleegkundig Specialist en Physician Assistant. Maastricht: Maastricht UMC+, RVE Patiënt \& Zorg, 2015.

12. Lambregts J. PVV, Grotendorst A. Beroepsprofiel verpleegkundig specialist. Verpleegkundigen \& Verzorgenden 2020, 2012.

13. The Nurse Practitioner in the Netherlands. Organisation DPNP,ed. 2015.

14. De Bruijn-Geraets DP, Van Eijk-Hustings YJL, Vrijhoef HJM. Evaluating newly acquired authority of nurse practitioners and physician assistants for reserved medical procedures in the Netherlands: a study protocol. J Adv Nurs 2014;70:2673-82.

15. von Elm E, Altman DG, Egger M, et al. STROBE Initiative. The Strengthening the Reporting of Observational Studies in Epidemiology (STROBE) Statement: guidelines for reporting observational studies. Int J Surg 2014;12:1495-9. 
16. Tong A, Sainsbury P, Craig J. Consolidated criteria for reporting qualitative research (COREQ): a 32-item checklist for interviews and focus groups. Int J Qual Health Care 2007;19:349-57.

17. Johnson RB, Onwuegbuzie AJ. Mixed Methods Research: A Research Paradigm Whose Time Has Come. Educational Researcher 2004;33:14-26.

18. Creswell JW, Plan Clark VL. Designing and Conducting Mixed Methods Research. Thousand Oaks, California: Sage Publications, 2006.

19. Aaltrichter $\mathrm{H}$, Feldman A, Posch P, et al. Teachers investigate their work; An introduction to action research across the professions. London 2008.

20. Onwuegbuzie AJ, Johnson RB. The Validity Issue in Mixed Research. Research in the Schools 2006;13:48-63.

21. Sidani S, Irvine D. A conceptual framework for evaluating the nurse practitioner role in acute care settings. J Adv Nurs 1999;30:58-66.

22. Sidani S, Doran DM, Mitchell PH. A theory-driven approach to evaluating quality of nursing care. J Nurs Scholarsh 2004;36:60-5.

23. Irvine D, Sidani S, Hall LM. Finding value in nursing care: a framework for quality improvement and clinical evaluation. Nurs Econ 1998;16:110-6.

24. Donabedian A. The quality of medical care: a concept in search of a definition. J Fam Pract 1979;9:277-84.

25. Grol R, Wensing M. Effective implementation: A model. In: Improving patient care: the implementation of change in clinical practice. London: Elsevier, 2005.

26. Scholten C, ljzerman MJ MA. Taakverschuiving van arts naar verpleegkundige. Maarssen: Elsevier/De Tijdstroom/LCVV, 1999.

27. de Bie J, Cuperus-Bosma JM, van der Jagt MA, et al. Risky procedures by nurses in hospitals: problems and (contemplated) refusals of orders by physicians, and views of physicians and nurses: a questionnaire survey. Int J Nurs Stud 2005;42:637-48.

28. Coles $\mathrm{E}$, Wells $\mathrm{M}$, Maxwell $\mathrm{M}$, et al. The influence of contextual factors on healthcare quality improvement initiatives: what works, for whom and in what setting? Protocol for a realist review. Syst Rev 2017;6:168.

29. De Bruijn-Geraets DP, Van Eijk-Hustings YJ, Vrijhoef HJ. Evaluating newly acquired authority of nurse practitioners and physician assistants for reserved medical procedures in the Netherlands: a study protocol. J Adv Nurs 2014;70:2673-82.

30. Crabtree BF, Miller WL, Research DQ. In: Publishing S, ed. Research Methods for Primary Care. Newbury Park CA 1992.

31. NVivo qualitative data analysis Software [program]. 10 version, 2012.

32. Guest G, Bunce A, Johnson L. How many interviews are enough? An experiment with data saturation and variability. Field Methods 2006;18:13

33. Zwijnenberg NC, Bours GJ. Nurse practitioners and physician assistants in Dutch hospitals: their role, extent of substitution and facilitators and barriers experienced in the reallocation of tasks. $J$ Adv Nurs 2012;68:1235-46.

34. Kroezen M, van Dijk L, Groenewegen PP, et al. Knowledge claims, jurisdictional control and professional status: the case of nurse prescribing. PLoS One 2013;8:e77279.

35. Kroezen M, Mistiaen P, van Dijk L, et al. Negotiating jurisdiction in the workplace: a multiple-case study of nurse prescribing in hospita settings. Soc Sci Med 2014;117:107-15.

36. Kroezen M, van Dijk L, Groenewegen PP, et al. Neutral to positive views on the consequences of nurse prescribing: Results of a national survey among registered nurses, nurse specialists and physicians. Int J Nurs Stud 2014;51:539-48.

37. Niezen MG, Mathijssen JJ. Reframing professional boundaries in healthcare: a systematic review of facilitators and barriers to task reallocation from the domain of medicine to the nursing domain. Health Policy 2014;117-151-69.

38. Maier CB, Aiken LH. Task shifting from physicians to nurses in primary care in 39 countries: a cross-country comparative study. Eur $J$ Public Health 2016;26:927-34.

39. Kroezen M, Francke AL, Groenewegen PP, et al. Nurse prescribing of medicines in Western European and Anglo-Saxon countries: a survey on forces, conditions and jurisdictional control. Int J Nurs Stud 2012;49:1002-12.

40. Kroezen M, van Dijk L, Groenewegen PP, et al. Nurse prescribing of medicines in Western European and Anglo-Saxon countries: a systematic review of the literature. BMC Health Serv Res 2011;11:127.

41. http://www.napa.nl/ (accessed 30 Jan 2018)

42. Martsolf GR, Auerbach DI, Arikfkhanova A. The Impact of Full Practice Authority for Nurse Practitioners and Other Advanced Practice Registered Nurses in Ohio, 2015.
43. Counsell SR, Callahan CM, Clark DO, et al. Geriatric care management for low-income seniors: a randomized controlled trial. JAMA 2007;298:2623-33.

44. Krichbaum K. GAPN postacute care coordination improves hip fracture outcomes. West J Nurs Res 2007;29:523-44.

45. Callahan CM, Boustani MA, Unverzagt FW, et al. Effectiveness of collaborative care for older adults with Alzheimer disease in primary care: a randomized controlled trial. JAMA 2006;295:2148-57.

46. Hooker RS. The Economic Basis of Physician Assistant Practice: An In-Depth Evaluation of the Cost-Effectiveness of Physician Assistants Physician Assistant, 2000:24;15.

47. Dillon D, Gary F. Full Practice Authority for Nurse Practitioners. Nurs Adm Q 2017;41:86-93.

48. Stange K. How does provider supply and regulation influence health care markets? Evidence from nurse practitioners and physician assistants. J Health Econ 2014;33:1-27.

49. Authority TDH. Advies Taakherschikking in de tweedelijns somatische zorg en curatieve geestelijke gezondheidszorg. Utrecht, 2012.

50. Ministry of of Health WaS. Kabinetsreactie advies NZa taakherschikking, CZ- 3132479: The Hague, 2012.

51. Authority TDH. Advies Integrale tarieven medisch specialistische zorg Utrecht, 2015.

52. https://www.aanp.org/legislation-regulation/state-legislation/statepractice-environment (accessed 30 Jan 2018).

53. Gadbois EA, Miller EA, Tyler D, et al. Trends in state regulation of nurse practitioners and physician assistants, 2001 to 2010. Med Care Res Rev 2015;72:200-19.

54. van Soeren M, Hurlock-Chorostecki C, Goodwin S, et al. The primary healthcare nurse practitioner in Ontario: a workforce study. Nurs Leadersh 2009;22:58-72.

55. Fornili KS, Fogger SA. Nurse Practitioner Prescriptive Authority for Buprenorphine: From DATA 2000 to CARA 2016. J Addict Nurs 2017;28:43-8.

56. Fong J, Buckley $\mathrm{T}$, Cashin $\mathrm{A}$, et al. Nurse practitioner prescribing in Australia: A comprehensive literature review. Aust Crit Care 2017;30.

57. Merkle F, Ritsema TS, Bauer S, et al. The physician assistant: Shifting the Paradigm of European medical practice? HSR Proc Intensive Care Cardiovasc Anesth 2011;3:255-62.

58. http://www.aspa-australianpas.org/about.htm (accessed 30 Jan 2018).

59. http://www.caopa.net/ (accessed 30 Jan 2018).

60. AAPA. https://www.aapa.org (accessed 30 Jan 2018).

61. Wiler JL, Ginde AA. State laws governing physician assistant practice in the United States and the impact on emergency medicine. J Emerg Med 2015;48-e49-e58.

62. Donald F, Martin-Misener R, Carter N, et al. A systematic review of the effectiveness of advanced practice nurses in long-term care. $J$ Adv Nurs 2013;69:2148-61.

63. Donald F, Kilpatrick K, Reid K, et al. Hospital to community transitional care by nurse practitioners: a systematic review of costeffectiveness. Int J Nurs Stud 2015;52:436-51.

64. Jennings N, Clifford S, Fox AR, et al. The impact of nurse practitioner services on cost, quality of care, satisfaction and waiting times in the emergency department: a systematic review. Int J Nurs Stud 2015;52:421-35.

65. Martin-Misener R, Harbman P, Donald F, et al. Cost-effectiveness of nurse practitioners in primary and specialised ambulatory care: systematic review. BMJ Open 2015;5:e007167.

66. Horrocks S, Anderson E, Salisbury C. Systematic review of whether nurse practitioners working in primary care can provide equivalent care to doctors. BMJ 2002;324:819-23.

67. Traczynski J, Udalova V. Nurse Practitioner Independence, Health Care Utilization, and Health Outcomes. Hawaii Uo, Ed. 2013.

68. Jalloh F, Tadlock MD, Cantwell S, et al. Credentialing and Privileging of Acute Care Nurse Practitioners to Do Invasive Procedures: A Statewide Survey. Am J Crit Care 2016;25:357-61.

69. Simcock P, Kingett B, Mann N, et al. A safety audit of the first 10000 intravitreal ranibizumab injections performed by nurse practitioners. Eye 2014;28:1161-4

70. DaCosta J, Hamilton R, Nago J, et al. Implementation of a nursedelivered intravitreal injection service. Eye 2014;28:734-40.

71. Norton L, Tsiperfal A, Cook K, et al. Effectiveness and Safety of an Independently Run Nurse Practitioner Outpatient Cardioversion Program (2009 to 2014). Am J Cardiol 2016;118:1842-6.

72. Ross AC, Polansky MN, Parker PA, et al. Understanding the role of physician assistants in oncology. $J$ Oncol Pract 2010;6:26-30.

73. Boodhoo L, Bordoli G, Mitchell AR, et al. The safety and effectiveness of a nurse led cardioversion service under sedation. Heart 2004;90:1443-6. 
74. Bhattacharyya M, Bradley $H$, Teherani A, et al. Nurse practitioner's knee injection clinics in the UK: The patient's perception. Journal of Orthopaedic Nursing 2005;9:134-9.

75. Newey M, Clarke M, Green T, et al. Nurse-led management of carpal tunnel syndrome: an audit of outcomes and impact on waiting times. Ann R Coll Surg Engl 2006;88:399-401.

76. Pfeifer UG, Schilling D. Non-Physician Endoscopy: How Far Can We Go? Visc Med 2016;32:13-20.
77. Duszak R, Walls DG, Wang JM, et al. Expanding roles of nurse practitioners and physician assistants as providers of nonvascular invasive radiology procedures. J Am Coll Radiol 2015;12:284-9.

78. Nyberg SM, Keuter KR, Berg GM, et al. Acceptance of physician assistants and nurse practitioners in trauma centers. JAAPA 2010;23:35-7.

79. Palinkas LA. Causality and Causal Inference in Social Work: Quantitative and Qualitative Perspectives. Res Soc Work Pract 2014;24:540-7. 